\title{
APTITUD COMBINATORIA DEL RENDIMIENTO Y SUS COMPONENTES EN DOS GRUPOS DE LÍNEAS DE MAÍZ ${ }^{1}$
}

\author{
César Guerrero-Guerrero ${ }^{2}$, Armando Espinoza-Banda², Arturo Palomo-Gil ${ }^{2}$,Emiliano Gutiérrez-del Río ${ }^{2}$, \\ Héctor Zermeño-González $z^{3}$,María P. González Castillo ${ }^{4}$
}

\section{RESUMEN}

Aptitud combinatoria del rendimiento y sus componentes en dos grupos de líneas de maíz. El objetivo del presente trabajo fue estimar en líneas de maíz los efectos de aptitud combinatoria general (ACG) y la aptitud combinatoria específica (ACE) de sus cruzas, para estimar su uso en un programa de hibridación. El presente trabajo se realizó en dos etapas, en el año 2006 la formación de cruzas en el campo experimental de la Universidad Autónoma Agraria Antonio Narro Unidad Laguna (UAAANUL), y la evaluación de las mismas en el año 2007, en dos localidades con tres ambientes; una localidad fue el campo experimental de la UAAANUL durante los ciclos primavera y verano, otra localidad fue el Niágara municipio de Aguascalientes, durante el ciclo de primavera. El material genético utilizado estuvo constituido por dos grupos de líneas: el primero lo formaron cuatro líneas provenientes de la UAAANUL, el segundo estuvo formado por diez líneas del Centro Internacional para el Mejoramiento de Maíz y Trigo (CIMMYT). Las estimaciones de la aptitud combinatoria general (ACG) y la aptitud combinatoria específica (ACE) fueron obtenidas usando un análisis de línea $\mathrm{x}$ línea. CML254 (10.88 t ha-1) y AN447 (6,64 t/ha) tuvieron el mayor efecto de ACG en ambos grupos. Los mayores efectos de ACE fueron para las cruzas CML264 x AN447 (128,8 t/ha) y CML247 x AN123 (116,2 t/ha). Estos resultados muestran que es posible estructurar un programa de híbridos, utilizando las mejores líneas del CIMMYT y de la UAAANUL.

Palabras clave: Zea mays L., híbridos, material genético, cruzas, ACE.

\begin{abstract}
Yield combining ability and components in two groups of inbred lines of maize. The objective of this work was to estimate the effects of the General Combining Ability (GCA) of inbreed lines, and Specific Combining Ability (SCA) of its crosses, and to evaluate its use in maize hybridization program. The present work was conducted in two stages; in year 2006 the crosses were done at the experimental station from Universidad Autónoma Agraria Antonio Narro Unidad Laguna (UAAANUL), and evaluation of the crosses was conducted in year 2007, at two localities and in three environments: UAAAN's experimental station during spring and summer cycles, Niagara and Aguascalientes Municipality during the spring cycle. The genetic material used were two groups of inbred lines, the first formed by four inbred lines from UAAANUL, and the second group was formed by ten inbred lines from Centro Internacional para el Mejoramiento del Maíz y Trigo (CIMMYT). The estimation of General Combining Ability (GCA) and Specific Combining Ability (SCA) were obtained using a test of line $\mathrm{x}$ line. CML254 (10.88 t/ha) and AN447 (6.64 t/ha) had the highest effects of GCA for both groups. The highest effects of SCA were for CML264 x AN447 (128.8 t/ha) and CML247 x AN123 (116.2 t ha1). These results show that is possible to structure a maize hybridization program, using the best inbred lines from CIMMYT and UAAANUL.
\end{abstract}

Key words: Zea mays L., hybrids, genetic material, crosses, SCA.

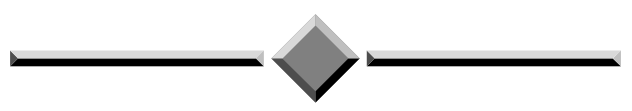

\footnotetext{
1 Recibido: 11 de octubre, 2010. Aceptado: 3 de octubre, 2011. Proyecto de investigación UAAANUL.

2 Departamento de Postgrado. Universidad Autónoma Agraria Antonio Narro Unidad Laguna, Periférico y Carretera a Santa Fe. Torreón, Coahuila. Tel. 01 (871) 729-7676, Fax 01 (871) 729-7610, Ext.7676. cesar_gg47@hotmail.com (Autor para correspondencia), aebanda7@ yahoo.com.mx, apalomog@mixmail.com, guredelrio@hotmail.com

3 Instituto Tecnológico de Torreón, Departamento de Agronomía. Carretera Torreón San Pedro Km 7.5. Torreón Coahuila, México Tel (871) 750-7198. zermegon@yahoo.com.mx

4 Instituto Politécnico Nacional, Unidad Durango. (CIIDIR). Sigma 119, Fracc. 20 de Noviembre Durango, Dgo. gcmary01@hotmail.com
} 


\section{INTRODUCCIÓN}

La necesidad de buscar nuevas alternativas para abaratar costos de producción principalmente del ganado lechero, hacen necesario realizar estudios en uno de los cultivos de mayor demanda como lo es el maíz forrajero, a fin de satisfacer las necesidades de la alimentación en la dieta de vacas lecheras, dada su alta productividad y calidad en verde y ensilado (LACTODATA 2011). Es importante buscar mejores alternativas en cuanto a genotipos que aseguren altos rendimientos de forraje tomando en cuenta una mayor relación hoja-tallo, elote-planta, alta producción de materia seca y mayor calidad nutritiva (Clark et al. 2002). De tal forma que al realizar ensilados, éstos presenten un alto valor nutritivo, lo que se verá reflejado en una mayor producción de leche, logrando de esta manera que una alta producción de forraje y de buen valor nutritivo abaraten costos de producción en la industria lechera, aumentando los dividendos de los productores (Rivas et al. 2006).

El éxito de cualquier programa genético con énfasis en el desarrollo de líneas endocriadas para la formación de híbridos, dependerá de la elección del germoplasma base a considerarse dentro del programa de mejoramientos (De León et al. 2005). Asimismo, el mejorador requiere de un conocimiento amplio del tipo de acción génica de los materiales en estudio, de los caracteres de mayor importancia económica tales como el alto potencial de rendimiento, buena aptitud combinatoria, producir buenas cruzas, entre otras. Lo anterior permitirá elegir el mejor esquema de mejoramiento que maximice la varianza genética, y de esta forma incrementar y fijar la frecuencia de genes favorables en la población (Navarro y Borrego 1993).

Cuando se está interesado en el mejoramiento de los híbridos resultantes del cruzamiento entre individuos de dos poblaciones, se recomienda conocer el comportamiento genético de las características de importancia económica de cada población, para elegir la estrategia de selección que permita obtener híbridos con mayores ventajas agronómicas (Vergara et al. 2005). Con la idea central de identificar una alternativa que mejore el comportamiento de los híbridos, derivados del cruzamiento entre líneas pertenecientes a los grupos tropical, subtropical húmedo y del trópico seco de México, en este trabajo, se planeó hacer un diseño genético de apareamiento entre dos grupos de líneas endogámicas, con el objeto de obtener información teórica de los efectos genéticos y tener la posibilidad de elegir los mejores híbridos (Chávez 1995). Al estimar los efectos de aptitud combinatoria general (ACG) y específica (ACE), nos permiten conocer los tipos de acción génica que controlan a los diferentes caracteres (Hallauer y Miranda 1988). Los efectos de ACG están relacionados con los genes de efectos aditivos de los progenitores, mientras que la ACE con los de dominancia y los epistáticos de las cruzas, confirmando e indicando la contribución genética diferencial en la expresión fenotípica, por lo que el desarrollo de líneas y la identificación de las mejores combinaciones híbridas con base en el potencial de rendimiento, determinan el éxito de un programa de mejoramiento genético (Antuna et al. 2003). Cuando en una población los efectos de aptitud combinatoria general (ACG), son más importantes que los efectos específicos (ACE), es recomendable mejorar a la población recurrente (Comstock y Robinson 1948). El mejoramiento genético por hibridación tendrá éxito si las dos o al menos una de las líneas de un híbrido son de alta ACG, condición que por sí misma asegura un alto rendimiento. Si adicionalmente el híbrido presenta un efecto positivo alto de ACE, su capacidad de rendimiento aumentará. En cambio, si las líneas son de baja ACG y su efecto de ACE es bajo, el rendimiento de la cruza será bajo (Reyes et al. 2004)

La decisión de qué diseño genético se deba emplear para conocer algunas propiedades genéticas de las poblaciones de interés, estará en función de los objetivos de la investigación. Por norma se debe optar por elegir el más práctico y sencillo, asegurando que proporcione la información requerida (Hallauer y Miranda 1988). Al respecto existen varias propuestas, entre las que destacan los diseños I, II y III de Carolina del Norte, generados por (Comstock y Robinson 1948) citados por (Márquez 1991).

Para este trabajo, se decidió utilizar el diseño II de Carolina del Norte con efectos fijos, por hacer cruzamientos entre dos poblaciones diferentes propuesto por (Comstock y Robinson 1948), y se ha utilizado con éxito en la estimación de los efectos de aptitud combinatoria general (ACG) de los individuos empleados como progenitores, y los efectos de aptitud combinatoria específica (ACE) de la progenie evaluada. El objetivo fue estimar los efectos de aptitud combinatoria general (ACG) y la aptitud combinatoria específica (ACE) de líneas de maíz y sus cruzas. 


\section{MATERIALES Y MÉTODOS}

El trabajo se realizó en dos partes, primero se formaron las cruzas en el ciclo agrícola primavera verano del año 2006, en el Campo Experimental de la Universidad Autónoma Agraria Antonio Narro Unidad Laguna, (UAAANUL) localizado geográficamente entre los paralelos $24^{\circ} 30^{\prime}$ y $27^{\circ}$ latitud Norte, y los meridianos $102^{\circ}$ y $104^{\circ} 40^{\prime \prime}$ longitud Oeste, con $1150 \mathrm{msnm}$ y un clima seco, caluroso, con temperatura media anual de $20-22^{\circ} \mathrm{C}$, precipitaciones escasas, con deficiencia de lluvias en todas sus estaciones y una precipitación media anual de $300 \mathrm{~mm}$, con régimen de lluvias en los meses de septiembre, octubre y noviembre, los vientos dominantes son alisios en dirección Sur, con velocidades de 27 - $44 \mathrm{~km} / \mathrm{h}$ (INEGI 2008).

La evaluación de las cruzas se realizó en el año 2007 en dos localidades y tres ambientes, la primera fue el campo experimental de la UAAANUL, una durante el ciclo de primavera y otra durante el ciclo de verano, la segunda localidad fue el ejido El Niágara, Municipio de Aguascalientes, Ags durante el ciclo de primavera.

El Ejido Niágara se ubica en la región occidental de la Altiplanicie Mexicana, en las coordenadas $21^{\circ}$ 53" de Latitud Norte, $102^{\circ} 18^{\prime \prime}$ de Longitud Oeste con altura de $1870 \mathrm{msnm}$, el clima es semiárido, con temperatura media anual de $17^{\circ} \mathrm{C}$, las temperaturas más altas se registran en los meses de abril, mayo y junio, y las más bajas en los meses de noviembre, diciembre, enero y febrero. La precipitación pluvial es de 526 $\mathrm{mm}$ al año, con lluvias abundantes en verano y poca intensidad el resto del año. Los vientos dominantes son alisios en dirección Sureste - Noroeste durante el verano y parte del otoño (INEGI 2008).

El material genético estudiado fueron dos grupos de líneas endogámicas: el primero, utilizado como probadores macho, estuvo formado por cuatro líneas cuyo origen proviene de la Universidad Autónoma Agraria Antonio Narro (UAAAN), las cuales se caracterizan por su adaptación a los climas del trópico seco, resistencia a plagas y resistencia a altas temperaturas ambientales. El segundo grupo, lo formaron diez líneas provenientes del Centro Internacional del Mejoramiento de Maíz y Trigo (CIMMYT), las cuales se caracterizan por su adaptación al clima tropical y subtropical húmedo, tolerancia al estrés de factores bióticos como resistencia a enfermedades y resistencia a la sequía (CIMMYT 1999):
M1; L-AN 447. Línea de ocho autofecundaciones, derivada de generaciones avanzadas del híbrido AN447 , con buena adaptabilidad.

M2; L-AN 130. Se derivó de la cuarta generación de la cruza de $\mathrm{H}-507$ x Celaya 2.

M3; L-AN 123. Formada a partir de una población criolla de la Región de los Altos, Mpio. de Concepción, Jalisco, que posee precocidad y tolerancia a sequía.

M4; L-AN 388P. Línea enana, con hojas anchas y verticales, generada a partir de la tercera generación del híbrido AN-388.

H1; CML-319 CIMMyT. RecyW89(Cr.Arg/CIM. ShPINPH)6-3-2-4-B-B.

H2; CML-264 Pob21 CIMMYT, POB21C5F2193-1-B- - -8-1-3-BBB-f.

H3; CML-254 Pob21 CIMMYT, TUXSEQ-1492-BBB- -1-BB-F.

H4; CML-313 CIMMYT, Pob501c0F6-3-3-2-1B-B.

H5; CML-273 Pob43 CIMMYT,(ACT643*43F7)2-3-2-1-bb-F.

H6; CML-247 Pool24 CIMMYT, (G24F119*G24F54)-6-4-1-1-BB-f.

H7; CML-271 Pob29 CIMMYT, pob29stec1hc256-4-1-_-BBB-F.

H8; CML-278 Pob43 CIMMYT, DMANTES8043-53-1-1-b-_-1-BB-f.

H9; CML-315 CIMMYT, Pob500P500c0F246-4$1-2-2-B * 3$.

H10; CML-318 Recy W87[B810(66)S3/G24S2]B-8-1-1-3-B*4.

La siembra se realizó en la primera localidad, el 18 de marzo, en el campo experimental de la UAAANUL durante el ciclo de primavera, y también el 21 de junio durante el ciclo de verano, la segunda localidad fue el ejido El Niágara municipio de Aguascalientes Ags, donde, se sembró el 24 de mayo durante el ciclo de primavera. Se utilizaron dos surcos de dos metros de longitud por parcela con distancia entre surcos de 0,75 $\mathrm{m}, \mathrm{y}$ un diseño experimental de bloques completos al azar con dos repeticiones. Treinta días después de la emergencia se dejaron seis plantas por metro lineal y $16,6 \mathrm{~cm}$ entre plantas, para obtener una densidad de población de 80000 plantas por hectárea.

Se aplicó la fórmula de fertilización 200 N, 90 P, $00 \mathrm{~K}$, en dos etapas: el $50 \%$ de nitrógeno y el total de fosforo en el momento de surcar, y el resto en la escarda 
a los 42 días después de la siembra. Se aplicaron cuatro riegos por gravedad; uno de presiembra y tres de auxilio procurando que las plantas no sufrieran por estrés durante las etapas del cultivo.

El control de maleza fue manual; se dieron dos deshierbes antes de la escarda y tres después del aporcado del cultivo. Las plagas que se presentaron fueron el gusano cogollero (Spodoptera frugiperda) y la pulga negra (Chaetocnema pulicaria), la primera se controló aplicando el insecticida Decís (Deltametrina), con dosis de 1,0 1/ha y en el caso de la pulga negra, se utilizó Lorsban (Insecticida organofosforado) a 1,0 1/ha.

Los cortes se hicieron a los 116 días después de la siembra, cuando el grano se encontraba en estado lechoso en ambas localidades con los tres ambientes, se tomaron tres plantas por parcela, primero se procedió a medir la altura de la planta, luego, se separaron los tres componentes principales del rendimiento en forraje verde, y se pesaron por separado: el tallo con la espiga, las hojas de la planta y el elote con hojas. Después se procedió a tomar las medidas del diámetro y longitud del elote, luego se procedió a sacar el promedio por planta en cada una de las características.

Las variables evaluadas fueron: rendimiento en forraje verde (RFV), peso del tallo (PT), peso de las hojas de la planta (PHP), peso del elote con hojas $(\mathrm{PECH})$, diámetro del elote (DE), longitud del elote (LE), altura de planta (AP). El análisis estadístico para las variables evaluadas, se realizó con el paquete estadístico SAS (SAS 1988), el análisis genético se realizó con el diseño II de apareamiento Carolina del Norte (Comstock y Robinson 1948) con efectos fijos, cuyo modelo lineal es:

$$
Y i j k=\mu+M i+H j+\Phi i j+€ i j k
$$

Donde, $\mathrm{i}=1,2, \ldots . \mathrm{m}$ (machos); $\mathrm{j}=1,2, \ldots . \mathrm{h}$ (hembras); $\mathrm{k}=1,2, . \mathrm{r}$ (rep); Yijk= observación de la cruza entre el i-esimo macho y la j-esima hembra en la kesima repetición; $\mu=$ Media general; $\mathrm{Mi}$ y $\mathrm{Hj}=$ Efecto del i-esimo macho y j-esima hembra; $\Phi \mathrm{ij}=$ Efecto de la interacción del i-esimo macho con la j-esima hembra; $€ \mathrm{ijk}=$ error experimental.

La estimación de los efectos de aptitud combinatoria general (ACG) para los machos y hembras, y la aptitud combinatoria específica (ACE) para las cruzas, se hizo según la propuesta de (Sprague y Tatum 1941), donde: $g i=\bar{Y}_{i .}-\bar{Y}_{. .} g_{j}=\bar{Y}_{. j}-\bar{Y}$

Sij= Yij - gi - gj $-\tilde{Y} \ldots$, y gi, gj y Sij son los efectos de ACG y ACE, respectivamente, para los imachos, las j-hembras y sus i j cruzas; $\tilde{Y}$ i. y $\tilde{Y}$.j son las medias de los machos y las hembras, Yij es el valor de la cruza ixj y $\tilde{Y}$.. es la media general de todas las ij cruzas.

\section{RESULTADOS Y DISCUSIÓN}

Los valores de los cuadrados medios, para localidades, en el análisis de varianza estimados, usando el diseño II de Carolina del Norte (Comstock y Robinson 1948) con efectos fijos, no muestran diferencias significativas para el rendimiento en forraje verde (RFV), ni para los principales componentes del rendimiento, lo que indica, que entre los tres ambientes de prueba no existen condiciones que permitan se diferencie un ambiente de otro. Los probadores macho (M) fueron significativos $(\mathrm{p} \leq 0,01)$ para todas las variables evaluadas, excepto, para rendimiento en forraje verde (RFV), que solo fue significativo al $(\mathrm{p} \leq 0,05)$, mostrando la gran diversidad genética que existe entre las líneas del grupo de la UAAANUL.

Para la interacción $\mathrm{H}$ x M, las cruzas mostraron diferencias significativas $(p \leq 0,01)$ en todas las variables evaluadas, lo cual podría deberse a la variabilidad genética que existe entre las líneas de ambos grupos, así como al diferente grado de combinación entre ellas. Para las interacciones L X M, L X H y L x H x $M$ no hubo significancia para ninguna de las variables evaluadas, en este caso no se detectó significancia para las interacciones genotipo $\mathrm{x}$ ambiente, de lo cual se deduce que las líneas tuvieron el mismo comportamiento en todas las localidades; este tipo de respuesta es consecuencia de un mejoramiento genético con poco avance, al estar expuesto solamente a los potenciales genotípicos, y omitir las bondades de la selección, para aquellos ambientes donde el efecto de la interacción es relevante (Moll et al. 1962). Cuando ocurren estas condiciones, aún es posible realizar el mejoramiento en una sola localidad, ya que las respuestas de la selección podrían ser expresadas en el resto de las localidades, lo que repercute en la eficiencia del proceso de evaluación. 
Las diferencias observadas, entre las líneas del CIMMYT y probadores líneas UAAAN, se deben a que provienen de diferente germoplasma y tienen diferente adaptación climática. Además por la diferencia en las condiciones climáticas entre ambas localidades y a las diferentes estaciones del año. El comportamiento diferencial de las variables RFV, PT, PHP, PECH, DE y LE a través de las cruzas de prueba desarrolladas, es atribuible a la gran diversidad genética prevaleciente en las líneas progenitoras, producto de su origen genético heterogéneo; lo cual ha sido también determinado por otros autores (De La Rosa et al. 2000, Soengas et al. 2003, De La Cruz et al. 2005). De igual manera, se han identificado híbridos de maíz de alto rendimiento, como resultado de la alta variabilidad genética entre las poblaciones de donde se derivaron las líneas, y a la diversidad genética entre las líneas probadoras, lo que indica la posibilidad de obtener híbridos cada vez más rendidores con calidad de forraje y grano (Moreno et al. 2002). La presencia de coeficientes de variación aceptables 11,$3 ; 11,7 ; 11,8$ y 15,8 en las variables AP, LE, DE y PHP, indicaron una precisión mayor en su muestreo; no así en las variables RFV, PT y PECH con valores de 19,$7 ; 21,9$ y $20,5 \%$, debido a que estas características son las más complicadas al momento de medir, por lo que se considera que estos valores están influenciados por errores al muestrear (Kang et al. 1999).

Las medias generales para cada variable, indicaron que al obtenerse un rendimiento de 93,4 t de forraje verde, el peso del tallo con espiga, el peso de las hojas de la planta y el peso del elote con hojas, contribuyeron con un 47,$31 ; 16,82$ y $35,86 \%$ respectivamente al rendimiento en forraje verde total aproximadamente; mientras que en otras investigaciones se obtuvieron 92 $\mathrm{t}$ de forraje verde, con el $63 \%$ de tallos y espigas, $15 \%$ de elote y $16 \%$ de hojas (Amador y Boschini 2000). Por otra parte, con híbridos de maíz adaptados a esta región, se han obtenido rendimientos similares a los encontrados en este estudio (Gutiérrez et al. 2002).

El comportamiento medio de las líneas progenitoras macho de la UAAANUL, mostró que dos líneas M1 (L-AN-447) 100,0 t/ha y M4 (L-AN-388P) 95,8 t/ ha, resultaron con los valores estadísticamente superiores para la mayoría de las variables evaluadas (Cuadro 1). Además, resultaron ser los progenitores macho con mayor valor de ACG para RFV, y para la mayoría de las variables evaluadas y los que intervinieron en las cruzas más rendidoras.

En el comportamiento medio de rendimiento en forraje verde de las líneas endogámicas progenitoras hembra del grupo del CIMMYT, mostró que H3 (CML-254) 104,3 t/ha, H1 (CML-319) 99,3 t/ha $y$ H5 (CML-273) 97,9 t/ha, resultaron con los valores promedio más altos, para el rendimiento en forraje verde $\mathrm{y}$ en los principales componentes del rendimiento (Cuadro 2), lo que muestra que en este grupo de líneas, casi todos los componentes contribuyeron al rendimiento, información que indica, uniformidad en el comportamiento de estos genotipos evaluados (López 2003). Los genotipos con menor rendimiento fueron los progenitores, tal como era de esperarse dado su grado de endogamia y los efectos que ésta tiene sobre la producción.

Cuadro 1. Comportamiento promedio de rendimiento en forraje verde en t/ha de las líneas endogámicas de maíz usadas como probadores macho, para siete características agronómicas. Combinado de dos localidades y tres ambientes, Torreón Coah y Aguascalientes Ags. México, 2007.

\begin{tabular}{cccccccc}
\hline & $\begin{array}{c}\text { Rendimiento } \\
\text { de forraje ver- } \\
\text { de (t/ha) }\end{array}$ & $\begin{array}{c}\text { Peso del tallo } \\
(\mathbf{t} / \mathbf{h a})\end{array}$ & $\begin{array}{c}\text { Peso de las } \\
\text { hojas de la } \\
\text { planta (t/ha) }\end{array}$ & $\begin{array}{c}\text { Peso del elote } \\
\text { con hojas } \\
(\mathbf{t} / \mathbf{h a})\end{array}$ & $\begin{array}{c}\text { Diámetro del } \\
\text { elote }(\mathbf{c m})\end{array}$ & $\begin{array}{c}\text { Longitud del } \\
\text { elote }(\mathbf{c m})\end{array}$ & $\begin{array}{c}\text { Altura de } \\
\text { planta }(\mathbf{m})\end{array}$ \\
\hline $\mathrm{M}_{1}$ & $100,0 \mathrm{a}$ & $49,2 \mathrm{a}$ & $16,7 \mathrm{a}$ & $34,0 \mathrm{ab}$ & $4,9 \mathrm{a}$ & $21,6 \mathrm{a}$ & $2,5 \mathrm{a}$ \\
$\mathrm{M}_{2}$ & $89,1 \mathrm{~b}$ & $42,7 \mathrm{~b}$ & $14,7 \mathrm{c}$ & $31,6 \mathrm{~b}$ & $4,9 \mathrm{a}$ & $19,5 \mathrm{c}$ & $2,3 \mathrm{~b}$ \\
$\mathrm{M}_{3}$ & $88,6 \mathrm{~b}$ & $40,9 \mathrm{~b}$ & $16,1 \mathrm{ab}$ & $31,5 \mathrm{~b}$ & $4,6 \mathrm{~b}$ & $20,6 \mathrm{~b}$ & $2,3 \mathrm{~b}$ \\
$\mathrm{M}_{4}$ & $95,8 \mathrm{ab}$ & $43,9 \mathrm{~b}$ & $15,2 \mathrm{bc}$ & $36,7 \mathrm{a}$ & $4,9 \mathrm{a}$ & $21,4 \mathrm{ab}$ & $2,1 \mathrm{c}$ \\
\hline
\end{tabular}

Medias con letras iguales no son estadísticamente diferentes (DMS, 0,05).

$\mathrm{M}=$ Machos. 
Cuadro 2. Promedio de rendimiento en forraje verde en t/ha de las líneas endogámicas de maíz usadas como hembras, para siete características agronómicas. Combinado de dos localidades y tres ambientes, Torreón Coah y Aguascalientes Ags. México, 2007.

\begin{tabular}{lccccccc}
\hline & $\begin{array}{c}\text { Rendimiento } \\
\text { de forraje ver- } \\
\text { de (t/ha) }\end{array}$ & $\begin{array}{c}\text { Peso del tallo } \\
(\mathbf{t} / \mathbf{h a})\end{array}$ & $\begin{array}{c}\text { Peso de las } \\
\text { hojas de la } \\
\text { planta (t/ha) }\end{array}$ & $\begin{array}{c}\text { Peso del elote } \\
\text { con hojas } \\
(\mathbf{t} / \mathbf{h a})\end{array}$ & $\begin{array}{c}\text { Diámetro del } \\
\text { elote }(\mathbf{c m})\end{array}$ & $\begin{array}{c}\text { Longitud del } \\
\text { elote }(\mathbf{c m})\end{array}$ & $\begin{array}{c}\text { Altura de la } \\
\text { planta }(\mathbf{m})\end{array}$ \\
\hline $\mathrm{H}_{1}$ & $99,3 \mathrm{ab}$ & $47,6 \mathrm{ab}$ & $16,7 \mathrm{ab}$ & $34,9 \mathrm{ab}$ & $4,86 \mathrm{ab}$ & $21,7 \mathrm{ab}$ & $2,34 \mathrm{ab}$ \\
$\mathrm{H}_{2}$ & $96,1 \mathrm{ab}$ & $46,6 \mathrm{ab}$ & $15,9 \mathrm{abc}$ & $33,5 \mathrm{ab}$ & $4,62 \mathrm{~b}$ & 19,8 & $2,39 \mathrm{a}$ \\
$\mathrm{H}_{3}$ & $104,3 \mathrm{a}$ & $49,8 \mathrm{a}$ & $17,8 \mathrm{a}$ & $36,5 \mathrm{a}$ & $4,95 \mathrm{ab}$ & $20,9 \mathrm{bcd}$ & $2,23 \mathrm{~b}$ \\
$\mathrm{H}_{4}$ & 89,1 & 41,8 & 13,9 & $33,4 \mathrm{ab}$ & $4,95 \mathrm{ab}$ & $20,8 \mathrm{bcd}$ & $2,32 \mathrm{ab}$ \\
$\mathrm{H}_{5}$ & $97,9 \mathrm{ab}$ & $45,7 \mathrm{abc}$ & $16,4 \mathrm{ab}$ & $35,7 \mathrm{a}$ & $4,98 \mathrm{a}$ & $20,9 \mathrm{bcd}$ & $2,28 \mathrm{ab}$ \\
$\mathrm{H}_{6}$ & 93,5 & $44,8 \mathrm{abc}$ & $16,3 \mathrm{ab}$ & $32,3 \mathrm{ab}$ & $4,98 \mathrm{a}$ & $20,5 \mathrm{bcd}$ & $2,35 \mathrm{ab}$ \\
$\mathrm{H}_{7}$ & 87,6 & 40,6 & 14,8 & $32,1 \mathrm{ab}$ & $4,78 \mathrm{ab}$ & 19,0 & $2,31 \mathrm{ab}$ \\
$\mathrm{H}_{8}$ & 82,2 & 38,8 & 13,9 & $29,5 \mathrm{~b}$ & $4,90 \mathrm{ab}$ & 20,1 & $2,32 \mathrm{ab}$ \\
$\mathrm{H}_{9}$ & $94,0 \mathrm{abc}$ & $45,9 \mathrm{abc}$ & $15,7 \mathrm{abc}$ & $32,2 \mathrm{ab}$ & $4,87 \mathrm{ab}$ & $21,3 \mathrm{abc}$ & $2,33 \mathrm{ab}$ \\
$\mathrm{H}_{10}$ & 89,9 & 39,8 & $15,3 \mathrm{bc}$ & $34,6 \mathrm{ab}$ & $4,94 \mathrm{ab}$ & $22,5 \mathrm{a}$ & $2,30 \mathrm{ab}$ \\
\hline
\end{tabular}

Medias con letras iguales no son estadísticamente diferentes (DMS, 0,05).

$\mathrm{H}=$ Hembras.

Los promedios de rendimiento en forraje verde (RFV) y sus componentes de las quince mejores cruzas $\left(\mathrm{M}^{*} \mathrm{H}\right)$, se pueden observar en el (Cuadro 3), donde se encontró que fluctúan entre 128,8 y 98,1 t/ha, valores que al promediarse dan una media de rendimiento en forraje verde igual a 93,4 t/ha. Las cruzas (2x1) CML-264 x L-AN-447 (128,8 t/ha), (9x1) CML-315 $x$ L-AN-447 (116,2 t/ha) y (1x4) CML-319 x L-AN$388 \mathrm{R}$, son las que resultaron con los valores promedio del rendimiento en forraje verde más altos, también lo fueron para las variables PT, PHP, PECH, DE, y AP.

La diversidad genética de los dos grupos de líneas, manifiesta que al combinar caracteres contrastantes, resulta una uniformidad en las cruzas y principalmente en los componentes del rendimiento, donde existe la posibilidad de que al conjuntar por recombinación y selección algunas líneas contrastantes, podrían resultar patrones heteróticos definidos. Los híbridos altamente rendidores obtienen su rendimiento no solamente de la heterosis, sino también, de factores hereditarios como la interacción múltiple de genes de tipo aditivo, por esto se debe conocer la importancia relativa de cada contribución genética en la producción de híbridos (Wong et al. 2006).

En los efectos de ACG de las líneas progenitoras, para machos, resaltan M1 (L-AN-447) 6,64 t/ha y M4
(L-AN-388R) 2,4 t/ha con valores positivos y significativos. El M1 para todas las variables evaluadas, en tanto que M4 para RFV, PECH, DE y LE (Cuadro 4). Respecto a las líneas progenitoras hembras o líneas del CIMMYT, resaltan H3 (CML-254) 10,88 t/ha, H1 (CML-319) 5,9 t/ha y H5 (CML-273) 4,49 t/ha para RFV, valores de ACG considerados como altos para ambos grupos de líneas según (Vergara et al. 2005). De acuerdo a los valores observados, se observan combinaciones específicas donde participan los machos M1 y M4, con las líneas hembras H3, H1 y H5, por lo que estas líneas deberán ser consideradas como hembras progenitoras potenciales para formar híbridos con las líneas progenitoras macho de la UAAANUL.

Algunos investigadores han confirmado el potencial genético de estas líneas con trabajos anteriores, señalando su valor genético en combinaciones híbridas. Los valores de ACG presentados por estos progenitores macho y hembras, presentaron una mayor contribución a la expresión fenotípica, lo que concuerda con lo encontrado por (De la Cruz et al. 2003). Con estos resultados secuenciales y acorde a las características estudiadas, pueden surgir resultados favorables para el mejoramiento del rendimiento (Márquez 1991). Al cruzar líneas de maíz endogámicas, para estudiar los tipos de aptitudes combinatorias, los comportamientos y 
Cuadro 3. Promedios de rendimiento en forraje verde en $\mathrm{t} / \mathrm{ha}$, de las 15 mejores cruzas de maíz para siete características agronómicas evaluadas. Combinado de dos localidades y tres ambientes, Torreón Coah y Aguascalientes Ags. México, 2007.

\begin{tabular}{cccccccc}
\hline Cruza & $\begin{array}{c}\text { Rendimiento } \\
\text { de forraje } \\
\text { verde (t/ha) }\end{array}$ & $\begin{array}{c}\text { Peso del tallo } \\
(\mathbf{t} / \mathbf{h a})\end{array}$ & $\begin{array}{c}\text { Peso de las } \\
\text { hojas de la } \\
\text { planta (t/ha) }\end{array}$ & $\begin{array}{c}\text { Peso del elote } \\
\text { con hojas } \\
(\mathbf{t} / \mathbf{h a})\end{array}$ & $\begin{array}{c}\text { Diámetro del } \\
\text { elote }(\mathbf{c m})\end{array}$ & $\begin{array}{c}\text { Longitud del } \\
\text { elote }(\mathbf{c m})\end{array}$ & $\begin{array}{c}\text { Altura de la } \\
\text { planta }(\mathbf{m})\end{array}$ \\
\hline $\mathbf{2}$ & $128,8 \mathrm{a}$ & $65,5 \mathrm{a}$ & $20,1 \mathrm{a}$ & $43,2 \mathrm{ab}$ & $5,2 \mathrm{a}$ & $22,7 \mathrm{a}$ & $2,8 \mathrm{a}$ \\
$9 \times 1$ & $116,2 \mathrm{ab}$ & $62,6 \mathrm{a}$ & $20,4 \mathrm{a}$ & $35,1 \mathrm{c}$ & $4,8 \mathrm{ab}$ & $22,3 \mathrm{a}$ & $2,6 \mathrm{a}$ \\
$1 \times 4$ & $115,9 \mathrm{ab}$ & $51,1 \mathrm{bc}$ & $17,8 \mathrm{ab}$ & $46,9 \mathrm{a}$ & $4,9 \mathrm{ab}$ & $22,5 \mathrm{a}$ & $2,1 \mathrm{~b}$ \\
$2 \times 2$ & $111,9 \mathrm{ab}$ & $56,0 \mathrm{ab}$ & 15,8 & $40,0 \mathrm{bc}$ & $5,1 \mathrm{a}$ & $20,3 \mathrm{ab}$ & $2,4 \mathrm{ab}$ \\
$3 \times 1$ & $110,4 \mathrm{ab}$ & $56,2 \mathrm{ab}$ & $19,1 \mathrm{ab}$ & $38,0 \mathrm{bc}$ & $4,8 \mathrm{ab}$ & $20,4 \mathrm{ab}$ & $2,2 \mathrm{ab}$ \\
$5 \times 1$ & $110,2 \mathrm{ab}$ & $53,5 \mathrm{~b}$ & $18,1 \mathrm{~b}$ & $38,5 \mathrm{bc}$ & $5,0 \mathrm{a}$ & $22,9 \mathrm{a}$ & $2,34 \mathrm{ab}$ \\
$6 \times 3$ & $109,6 \mathrm{abc}$ & $50,5 \mathrm{~b}$ & $20,3 \mathrm{a}$ & $38,6 \mathrm{bc}$ & $4,7 \mathrm{ab}$ & $21,2 \mathrm{a}$ & $2,5 \mathrm{ab}$ \\
$8 \times 1$ & $109,2 \mathrm{abc}$ & $57,5 \mathrm{ab}$ & $16,4 \mathrm{c}$ & $35,2 \mathrm{c}$ & $4,9 \mathrm{ab}$ & $19,3 \mathrm{ab}$ & $2,7 \mathrm{a}$ \\
$4 \times 4$ & $107,9 \mathrm{abc}$ & $47,3 \mathrm{~b}$ & 15,5 & $45,0 \mathrm{a}$ & $4,8 \mathrm{ab}$ & $21,6 \mathrm{a}$ & $2,1 \mathrm{~b}$ \\
$3 \times 3$ & $104,7 \mathrm{bc}$ & 49,0 & $19,4 \mathrm{ab}$ & $36,2 \mathrm{c}$ & $5,0 \mathrm{a}$ & $21,2 \mathrm{a}$ & $2,3 \mathrm{ab}$ \\
$5 \times 2$ & $102,6 \mathrm{bc}$ & $49,8 \mathrm{c}$ & $18,2 \mathrm{ab}$ & $34,5 \mathrm{c}$ & $4,7 \mathrm{ab}$ & $18,6 \mathrm{ab}$ & $2,3 \mathrm{ab}$ \\
$3 \times 2$ & $101,5 \mathrm{bc}$ & 48,7 & $16,4 \mathrm{bc}$ & $36,4 \mathrm{c}$ & $5,0 \mathrm{a}$ & $20,7 \mathrm{a}$ & $2,3 \mathrm{ab}$ \\
$10 \times 4$ & $100,4 \mathrm{~cd}$ & $44,2 \mathrm{a}$ & $15,6 \mathrm{ab}$ & $40,6 \mathrm{bc}$ & $4,9 \mathrm{ab}$ & $22,2 \mathrm{a}$ & $2,0 \mathrm{bc}$ \\
$1 \times 3$ & $99,1 \mathrm{~cd}$ & 47,1 & $18,8 \mathrm{ab}$ & 33,1 & $4,8 \mathrm{ab}$ & $21,8 \mathrm{a}$ & $2,1 \mathrm{~b}$ \\
$9 \times 4$ & 98,1 & 48,7 & 14,4 & $34,9 \mathrm{c}$ & $5,1 \mathrm{a}$ & $22,0 \mathrm{a}$ & $2,1 \mathrm{~b}$ \\
\hline
\end{tabular}

Medias con letras iguales no son estadísticamente diferentes (DMS, 0,05).

+ Hembras, ổ Machos.

valores dependerán de la constitución genética de cada uno de los progenitores y de la diversidad de su origen (Sprague y Tatum 1942).

Los efectos de aptitud combinatoria específica (ACE) se presentan en el (Cuadro 5), donde se observó, que dentro de las mejores 15 cruzas presentadas, las cruzas 2x1 (CML-264 x L-AN-447) 26,0 t/ha, 6x3 (CML-247 x L-AN-123) 20.79 t/ha, 8x1 (CML-278 x L- AN-447) 20,29 t/ha y 2x2 (CML-264 x L-AN130) $20,02 \mathrm{t} / \mathrm{ha}$, resultaron con los valores más altos y positivos de ACE para RFV y en los principales componentes del rendimiento. En general se encontró, que líneas con altos valores de ACG formaron las cruzas con mayor expresión de ACE y de rendimiento, coincidiendo con Wong et al (2006). Por lo tanto, es factible identificar las mejores combinaciones de líneas en híbridos para alta productividad (para ACG), probar y seleccionar combinaciones híbridas únicas donde se expresen altos valores de ACE, aun cuando la alta ACG haya sido la más importante para la identificación de las líneas (Hallauer y Miranda 1988).

La contribución a la variación entre las cruzas para la mayoría de las características evaluadas, es atribuible al efecto no aditivo de la interacción Macho $\mathrm{x}$ Hembra $(\mathrm{MxH})$, ya que los valores de ACE fueron entre dos y tres veces superiores a los de ACG, coincidiendo con lo reportado por (De la Cruz et al. 2005), quienes encontraron mayores valores para $\mathrm{ACE}$ que para ACG, sin embargo, (De la Rosa et al. 2000), reportaron diferencias significativas para ambas fuentes de variación ACG y ACE. Deberá también analizarse la calidad del forraje para elote, tallos, hojas y vainas del tallo de cada hoja, para maximizar y complementar los avances en la investigación en maíces forrajeros, donde se considere el incremento del rendimiento, calidad de la planta y el grano (Moreno et al. 2000). (Argillier et al. 2000) encuentran que la ACG de las líneas, es la fuente de variación más importante para 
Cuadro 4. Aptitud Combinatoria General (ACG) de líneas de maíz usadas como machos y hembras para rendimiento de forraje verde, en siete características evaluadas. Combinado de dos localidades y tres ambientes, Torreón Coah y Aguascalientes Ags. México. 2007.

\begin{tabular}{cccccccc}
\hline Padre & $\begin{array}{c}\text { Rendimiento } \\
\text { de forraje ver- } \\
\text { de (t/ha) }\end{array}$ & $\begin{array}{c}\text { Peso del tallo } \\
\mathbf{( t / h a )}\end{array}$ & $\begin{array}{c}\text { Peso de las } \\
\text { hojas de la } \\
\text { planta (t/ha) }\end{array}$ & $\begin{array}{c}\text { Peso del elote } \\
\text { con hojas } \\
(\mathbf{t} / \mathbf{h a})\end{array}$ & $\begin{array}{c}\text { Diámetro del } \\
\text { elote (cm) }\end{array}$ & $\begin{array}{c}\text { Longitud del } \\
\text { elote (cm) }\end{array}$ & $\begin{array}{c}\text { Altura de la } \\
\text { planta (m) }\end{array}$ \\
\hline M1 & $6,64^{*}$ & $5,07^{* *}$ & 1 & 0,56 & 0,05 & $0,82^{*}$ & $0,19^{*}$ \\
M2 & $-4,287$ & $-1,47$ & -1 & $-1,82$ & 0,07 & $-1,28$ & $-0,01$ \\
M3 & $-4,73$ & $-3,22$ & $0,47^{*}$ & $-1,98$ & $-0,21$ & $-0,16$ & 0,02 \\
M4 & 2,4 & $-0,4$ & $-0,45$ & $3,25^{*}$ & $0,11^{*}$ & 0,63 & $-0,21$ \\
H1 & $5,9 *$ & $3,42^{*}$ & $1,05^{*}$ & 1,42 & $-0,02$ & 0,94 & 0,02 \\
H2 & 2,74 & $2,49^{*}$ & 0,21 & 0,04 & $-0,26$ & $-0,94$ & 0,07 \\
H3 & $10,88^{* *}$ & $5,64^{* *}$ & $2,18^{* *}$ & $3,06^{*}$ & $0,07^{*}$ & 0,11 & $-0,09$ \\
H4 & $-4,3$ & $-2,38$ & $-1,75$ & $-0,17$ & $0,07^{*}$ & 0,08 & 0 \\
H5 & $4,49 *$ & $1,51^{*}$ & 0,71 & $2,27 *$ & 0,1 & 0,19 & $-0,04$ \\
H6 & 0,12 & 0,7 & $0,59^{*}$ & $-1,17$ & 0,1 & $-0,24$ & 0,03 \\
H7 & $-3,75$ & $-3,54$ & $-0,88$ & $-1,33$ & $-0,1$ & $-1,72$ & $-0,01$ \\
H8 & $-11,15$ & $-5,37$ & $-1,78$ & -4 & 0,02 & $-0,69$ & 0 \\
H9 & 0,62 & $1,8 *$ & 0,04 & $-1,22$ & $-0,01$ & 0,57 & 0,01 \\
H10 & $-3,52$ & $-4,32$ & $-0,33$ & 1,13 & 0,06 & $1,73^{* *}$ & $-0,02$ \\
\hline
\end{tabular}

$* \mathrm{y} * *=$ Efectos positivos y mayores de cero.

$\mathrm{M}=$ Machos, $\mathrm{H}=$ Hembras.

características de rendimiento en forraje del maíz, lo que refuerza la idea de seleccionar para rendimiento y calidad del forraje.

En el presente trabajo, resultaron algunos híbridos superiores que en un futuro se podrían explotar comercialmente, como son las cruzas; 2x1 (CML-264 x L-AN-447), 9x1 (CML-315 x L-AN-447), 1x4 (CML319 x L-AN-388R) y 2x2 (CML-264 x L-AN-130), por lo que es necesario sembrarlos en parcelas demostrativas para que los agricultores los identifiquen y los comparen con los híbridos que ellos siembran.

El grado de heterosis, con relación al progenitor medio (h) y al progenitor superior (h') para cada una de las variables evaluadas, se presentan en el (Cuadro 6), donde se puede observar que el valor máximo y mínimo de heterosis, para rendimiento de forraje verde (RFV) con relación al progenitor medio, fueron de 31,3 a $3,3 \%$, que corresponden a las cruzas $2 \times 1$ y 9x4, respectivamente. En la heterosis referente al mejor progenitor, los valores máximo y mínimo fueron de $28,7 \%$ para la cruza $2 \times 1$ y de $-2,7 \%$ para la cruza $3 \times 2$. $\mathrm{Al}$ respecto, en fitomejoramiento, se considera que el nivel deseable para el aprovechamiento de la heterosis en una cruza, es de cuando menos el 20\% (De la Cruz et al. 2003).

La mayor expresión heterótica con respecto al progenitor medio y al mayor progenitor, ocurrió en la cruza 2x1, formada por la hembra H2 (CML-264) y el macho M1 (L-AN-447), cuyos orígenes son grupos de líneas no emparentadas, por lo que difieren en su origen genético. Sin embargo existe un grupo emparentado, formado por los progenitores M2 (LAN-130) y M3 (L-AN-123), que al cruzarse con las líneas H1 (CML-319) y H3 (CML-254), dieron valores bajos en casi todas las variables. El grado de heterosis resultante en los mejores progenitores, sugiere suficiente variabilidad genética entre las poblaciones, mostrando que los loci homocigotos 
Cuadro 5. Aptitud combinatoria específica (ACE) de las 15 mejores cruzas de maíz según su capacidad de rendimiento en forraje verde, para siete características agronómicas evaluadas. Combinado de dos localidades y tres ambientes, Torreón Coah y Aguascalientes Ags. México, 2007.

\begin{tabular}{cccccccc}
\hline Cruza & $\begin{array}{c}\text { Rendimiento } \\
\text { de forraje } \\
\text { verde (t/ha) }\end{array}$ & $\begin{array}{c}\text { Peso del tallo } \\
(\mathbf{t} / \mathbf{h a})\end{array}$ & $\begin{array}{c}\text { Peso de las } \\
\text { hojas de la } \\
\text { planta (t/ha) }\end{array}$ & $\begin{array}{c}\text { Peso del elote } \\
\text { con hojas } \\
(\mathbf{t} / \mathbf{h a})\end{array}$ & $\begin{array}{c}\text { Diámetro del } \\
\text { elote }(\mathbf{c m})\end{array}$ & $\begin{array}{c}\text { Longitud del } \\
\text { elote }(\mathbf{c m})\end{array}$ & $\begin{array}{c}\text { Altura de la } \\
\text { planta }(\mathbf{m})\end{array}$ \\
\hline $2 \times 1$ & $26,0^{* *}$ & $13,75^{* *}$ & $3,22^{* *}$ & $9,12^{* *}$ & $0,53^{*}$ & $2,09^{*}$ & $0,25^{*}$ \\
$9 \times 1$ & $15,52^{*}$ & $11,61^{* *}$ & $3,67^{* *}$ & 2,29 & $-0,09$ & 0,13 & 0,13 \\
$1 \times 4$ & $14,18^{*}$ & 3,98 & 1,55 & $8,75^{* *}$ & $-0,06$ & 0,2 & 0,01 \\
$2 \times 2$ & $20,02^{* *}$ & $10,84^{*}$ & 0,9 & $8,35^{* *}$ & $0,41^{*}$ & $1,76^{*}$ & 0,07 \\
$3 \times 1$ & $-0,54$ & 1,36 & 0,28 & 0,92 & $-0,2$ & $-1,27$ & $-0,17$ \\
$5 \times 1$ & 5,65 & 2,77 & 0,74 & 2,19 & 0,0 & $1,15^{*}$ & $-0,13$ \\
$6 \times 3$ & $20,79 * *$ & $8,89 *$ & $-6,4$ & $8,31^{* *}$ & $-0,01$ & 0,89 & $0,22^{*}$ \\
$8 \times 1$ & $20,29 * *$ & $13,68^{* *}$ & 1,53 & $5,14 *$ & $-0,05$ & $-1,57$ & $0,21^{*}$ \\
$4 \times 4$ & $16,38^{*}$ & 5,94 & $2,0 *$ & $8,46^{* *}$ & $-0,25$ & 0,16 & 0,01 \\
$3 \times 3$ & 5,13 & 2,42 & 1,1 & 1,69 & 0,31 & 0,47 & 0,13 \\
$5 \times 2$ & 8,977 & 5,65 & $2,78^{*}$ & 0,62 & $-0,27$ & $-1,05$ & 0,06 \\
$3 \times 2$ & 1,487 & 0,36 & $-0,49$ & 1,7 & 0,04 & $1,14^{*}$ & 0,13 \\
$10 \times 4$ & 8,1 & 4,73 & 0,69 & 2,76 & $-0,09$ & $-0,89$ & $-0,05$ \\
$1 \times 3$ & 4,51 & 2,79 & 1,61 & 0,15 & 0,16 & 0,29 & $-0,19$ \\
$9 \times 4$ & 1,66 & 3,19 & $-0,81$ & $-0,63$ & 0,15 & 0,06 & $-0,02$ \\
\hline
\end{tabular}

$* \mathrm{y} * *=$ Efectos positivos y mayores de cero. + Hembras, $\partial$ Machos. $(*=\mathrm{P} \leq 0,05, * *=\mathrm{P} \leq 0,01)$.

fueron importantes en explicar la variabilidad genética existente para el rendimiento de forraje verde y sus componentes (Espinoza et al. 2003). Se observaron importantes combinaciones heteróticas entre las cruzas de las líneas de CIMMYT usadas como hembras con las líneas de la UAAANUL usadas como probadores, las cuales pueden ser explotadas para el desarrollo de nuevos híbridos comerciales en un corto tiempo.

\section{LITERATURA CITADA}

Amador, AL; Boschini, C. 2000. Fenología productiva y nutricional de maíz para la producción de forraje. Agronomía Mesoamericana 11(1):171-177.

Antuna, GO; Rincón, SF; Gutiérrez, RE; Ruiz, TNA; Bustamante, GL. 2003. Componentes genéticos de caracteres agronómicos y de calidad fisiológica de semillas en líneas de maíz. Rev. Fitotec. Mex. Vol. 26(1):11-17.

Argillier, O; Méchin, V; Barriére, Y. 2000. Inbred line evaluation and breeding for digestibility-related traits in forage maize. Crop Science 40:1596-1600.

Chávez, JL. 1995. Mejoramiento de plantas 1. 2 ed. Ed. Trillas. México. 136 p.

CIMMYT (Centro Internacional del Mejoramiento de Maíz y Trigo). 1999. Maize inbred lines relased by CIMMYT. A compilation of 424 CIMMYT maize lines (CMLs). First draft. México, D.F. 56 p.

Clark, PW; Kelm, S; Endres, ML. 2002. Effect of feeding a corn hybrid selected for leafiness as silage or grain to lactating dairy cattle. J. dairy Sci. 85:607-612.

Comstock, RE; Robinson, HF. 1948. The components of genetic variance in populations of biparental progenies 
Cuadro 6. Heterosis en base al progenitor medio (h) y al mejor progenitor (h'), para los principales componentes del rendimiento de las cruzas directas. Combinado de dos localidades y tres ambientes, Torreón Coah y Aguascalientes Ags. México, 2007.

\begin{tabular}{|c|c|c|c|c|c|c|c|c|c|c|c|c|c|c|}
\hline \multirow[t]{2}{*}{$\begin{array}{c}\text { Cruza } \\
q \delta\end{array}$} & \multicolumn{2}{|c|}{$\begin{array}{c}\text { Rendimiento } \\
\text { de forraje } \\
\text { verde } \\
\text { (t/ha) } \\
\end{array}$} & \multicolumn{2}{|c|}{$\begin{array}{l}\text { Peso del tallo } \\
\text { (t/ha) }\end{array}$} & \multicolumn{2}{|c|}{$\begin{array}{c}\text { Peso de las } \\
\text { hojas de la } \\
\text { planta } \\
\text { (t/ha) }\end{array}$} & \multicolumn{2}{|c|}{$\begin{array}{l}\text { Peso del elote } \\
\text { con hojas } \\
\text { (t/ha) }\end{array}$} & \multicolumn{2}{|c|}{$\begin{array}{c}\text { Diámetro del } \\
\text { elote } \\
(\mathrm{cm})\end{array}$} & \multicolumn{2}{|c|}{$\begin{array}{l}\text { Longitud del } \\
\text { elote } \\
\text { (cm) }\end{array}$} & \multicolumn{2}{|c|}{$\begin{array}{c}\text { Altura de la } \\
\text { planta } \\
(\mathbf{m})\end{array}$} \\
\hline & $\mathrm{h}$ & h' & $\mathrm{h}$ & $h^{\prime}$ & $\mathrm{h}$ & h' & $\mathrm{h}$ & h' & $\mathrm{h}$ & h' & $\mathrm{h}$ & h' & $\mathrm{h}$ & h' \\
\hline $2 \times 1$ & 31,3 & 28,7 & 36,5 & 33 & 23,4 & 20,5 & 27,9 & 26,9 & 8,9 & 5,5 & 9,8 & 5,3 & 15,5 & 12,7 \\
\hline $9 \times 1$ & 19,7 & 16,1 & 31,6 & 27,2 & 25,8 & 22,2 & 5,9 & 3,1 & $-1,4$ & $-2,0$ & 3,8 & 3,2 & 9,5 & 5,6 \\
\hline $1 \times 4$ & 18,8 & 16,7 & 12 & 7,5 & 11,6 & 6,6 & 30,9 & 27,7 & $-0,3$ & $-1,6$ & 4,6 & 3,8 & $-3,8$ & $-8,5$ \\
\hline $2 \times 2$ & 30,8 & 27,4 & 25,4 & 20,1 & 3,3 & $-0,6$ & 22,9 & 19,5 & 6,6 & 3,0 & 3,3 & 2,4 & 4,3 & 2,5 \\
\hline $3 \times 1$ & 8 & 5,8 & 13,6 & 12,9 & 10,8 & 7,2 & 7,7 & 4 & $-2,8$ & $-3,0$ & $-3,8$ & $-2,2$ & $-5,1$ & $-10,4$ \\
\hline $5 \times 1$ & 11,3 & 10,1 & 12,8 & 8,7 & 9,6 & 8,7 & 10,3 & 7,7 & 1,5 & 1,0 & 7,8 & 9,4 & $-2,3$ & $-6,8$ \\
\hline $6 \times 3$ & 27,9 & 24,2 & 17,8 & 12,6 & $-36,1$ & $-36,4$ & 21,1 & 19,6 & $-1,3$ & $-4,4$ & 3,4 & 3,2 & 10,4 & 10,2 \\
\hline $8 \times 1$ & 19,8 & 9,1 & 30,7 & 16,9 & 7,4 & $-1,5$ & 10,8 & 3,3 & $-0,3$ & $-0,6$ & $-7,2$ & $-10,5$ & 12,6 & 8,4 \\
\hline $4 \times 4$ & 16,7 & 12,6 & 10,6 & 8,1 & 6,2 & 1,6 & 28,5 & 22,6 & $-3,2$ & $-3,6$ & 2,4 & 3,8 & $-4,3$ & $-8,6$ \\
\hline $3 \times 3$ & 8,5 & 0,4 & 8 & $-1,6$ & 14,2 & 8,8 & 6,5 & $-0,8$ & 5 & 2,0 & 2,1 & 1,5 & 4,2 & 1,7 \\
\hline $5 \times 2$ & 9,7 & 4,8 & 12,8 & 9,1 & 16,9 & 10,8 & 2,5 & $-3,4$ & $-3,7$ & $-4,0$ & $-7,9$ & $-11,1$ & 1,5 & 0,9 \\
\hline $3 \times 2$ & 4,9 & $-2,7$ & 5,3 & $-2,2$ & 0,6 & $-8,3$ & 6,8 & $-0,3$ & 2,2 & 2,2 & 2,7 & $-0,7$ & 3,5 & 1,7 \\
\hline $10 \times 4$ & 8,1 & 4,8 & 5,7 & 0,9 & 2 & 1,6 & 13,9 & 10,6 & $-0,1$ & $-0,6$ & 1,3 & $-1,2$ & $-7,5$ & $-11,3$ \\
\hline $1 \times 3$ & 5,4 & $-0,2$ & 6,5 & $-0,9$ & 14,4 & 12,4 & $-0,4$ & $-5,2$ & 0,9 & $-1,0$ & 3,2 & 0,6 & $-7,3$ & $-7,3$ \\
\hline $9 \times 4$ & 3,3 & 2,4 & 8,7 & 6,1 & $-6,5$ & -8 & 1,1 & -5 & 4,1 & 2,8 & 3,1 & 2,9 & $-5,4$ & $-9,9$ \\
\hline
\end{tabular}

+ Hembras, $\delta^{\wedge}$ Machos. $\mathrm{h}=$ heterosis en base al progenitor medio, $\mathrm{h}$ '= heterosis en base al progenitor superior.

and their use in estimating the average degree of dominance. Biometrics 4:254-266.

De La Cruz, LE; Gutiérrez, RE; Palomo, GA; Rodríguez, HS. 2003. Aptitud combinatoria y heterosis de líneas de maíz en la Comarca Lagunera. Revista Fitotecnia Mexicana 26(4):279-284.

De La Cruz, E; Rodríguez, S; Estrada, M; Mendoza, J; Brito, N. 2005. Análisis dialélico de líneas de maíz QPM para características forrajeras. Universidad y Ciencia 21(41):19-26.

De León, CH; Rincón, SF; Reyes, VH; Samano, GD; Martínez, ZG; Cavazos, CR; Figueroa, CJ. 2005. Potencial de rendimiento y estabilidad de combinaciones germoplásmicas formadas entre grupos de maíz. Revista Fitotecnia Mexicana 28(2):135-143.

De La Rosa, A; De León, H; Martínez, G; Rincón, F. 2000. Heterosis, habilidad combinatoria y diversidad genética en híbridos comerciales de maíz (Zea mays L.). Agronomía Mesoamericana 11(1):113-122.

Espinoza, BA; Gutiérrez, RE; Palomo, GA; García, JL. 2003. Efectos genéticos en cruzas entre híbridos comerciales de maíz forrajero. Revista Fitotecnia Mexicana 25(2):171-178.

Gutiérrez, RE; Palomo, GA; Espinoza, BA; Lázaro, E. 2002. Aptitud combinatoria y heterosis para rendimiento de línea de maíz en la Comarca Lagunera. Revista Fitotecnia Mexicana 25:271-277.

Hallauer, RA; Miranda, FO. 1988. Quantitative genetics in maize breeding. The Iowa State University Press Ames, Iowa, USA. 468 p.

INEGI (Instituto Nacional de Geografía y Estadística). 2008. Atlas nacional interactivo de México. (en línea). Estado de Coahuila. Estado de Aguascalientes. Consultado 16 mayo 2010. Disponible en http://www.inegi.org.mx 
Kang, SM; Kushairi, DA; Zhang, Y; Magari, R. 1999. Combining ability for rind puncture resistance in maize. Crop Science 39:368-371.

LACTODATA. 2011. Información sobre el sector lechero en México. Consultado 30 sept. 2011. Disponible http:// www.lechemexico.org.mx.

López, LM. 2003. El cultivo de maíz en México y la contribución del fitomejorador para favorecer la autosuficiencia. Universidad Autónoma Agraria Antonio Narro Unidad Laguna. México. Revista Mexicana de Agronegocios 12:596-605.

Márquez, SF. 1991. Geotecnia vegetal. Métodos y Teoría. Tomo III. AGT Editor. S. A. México, D.F. 500 p.

Moll, RH; Salhuana, WS; Robinson, HF. 1962 Heterosis and genetic diversityin varieties of maize. Crop Science 2:197-198.

Moreno, GJ; Martínez, I; Brichette, I; López, A; Castro, P. 2000. Breeding potential of European flint and U.S. Corn belt dent maize populations for forage use. Crop Science 40:1588-1595.

Moreno, PE; Lewis, CDB; Cervantes, T; Torres, JL. 2002. Aptitud combinatoria de líneas de maíz de Valles Altos en suelos con alto y bajo contenido de nitrógeno. Revista Fitotecnia Mexicana 25: 253-259.

Navarro, GE; Borrego, FE. 1993. Efectos génicos y heterosis en poblaciones parentales y poblaciones derivadas de maíz (Zea mays L). Agronomía Mesoamericana. 4:7-10.

Peña, RA; Núñez, GH; González, FC. 2003. Importancia de la planta y el elote en poblaciones de maíz para el mejoramiento genético de la calidad forrajera. Téc. Pecu. Méx. 41(1):63-74.

Reyes, LD; Molina, GJD; Oropeza, RMA; Moreno, PEC. 2004. Cruzas dialélicas entre líneas autofecundadas de maíz derivadas de la raza tuxpeño. Rev. Fitotec. Mex. 27(1):49-56.

Rivas, M; Carballo, A; Perez, J; Serrano, JG; García, ZA. 2006. Rendimiento y calidad de ensilado de seis genotipos de maíz cosechados en dos estados de madurez. Memoria XVIII Reunión científica tecnológica forestal y agropecuaria. Veracruz. p. 463470 .

Soengas, P; Ordás, B; Malvar, RA; Revilla, P; Ordás, A. 2003. Heterotic patterns among flint maize populations. Crop Science 43:844-849.

Sprague, GF; Tatum, LA. 1941. General versus specific combining ability in single crosses of corn. J. Amer. Soc. Agron. 34:923-932.

SAS (Statistical Analysis System). 1988. User's Guide. Version 6.11. SAS Institute Inc. Cary, N.C., USA.

Vergara, AN; Rodríguez, HS; Córdova, OHS. 2005. Aptitud combinatoria general y específica de líneas de maíz (Zea mays) tropical y subtropical. Agronomía Mesoamericana 16(2):137-143.

Wong, RR; Gutiérrez, RE; Rodríguez, HS; Palomo, GA; Córdoba, OH; Espinoza, BA. 2006. Aptitud combinatoria y parámetros genéticos de maíz para forraje en la Comarca Lagunera, México. Revista Universidad y Ciencia 22(2):16-24. 